\title{
Is Rett Syndrome a Subtype of Pervasive Developmental Disorders? ${ }^{1}$
}

\author{
Luke Y. Tsai \\ Child and Adolescent Psychiatry Service, Department of Psychiatry, \\ University of Michigan Medical Center
}

The author reviews the issue on whether Rett syndrome (RS) is a subtype of pervasive developmental disorders (PDDs). More than 200 articles of $R S$ have been published in the last 10 years. Internal and external validities of $R S$ have been established by several independent studies. There remains the question whether RS presents clinical features that meet the total criteria for PDDs. The available data seem to support the idea of classifying RS as a subtype of PDDs in the DSM-IV.

Rett syndrome (RS) is a condition characterized by a specific developmental course, characteristic neurological features, and a variety of unusual behaviors and developmental findings. In the DSM-III-R diagnostic scheme (American Psychiatric Association [APA], 1987), RS and other pervasive developmental disorders (PDDs) such as atypical autism and disintegrative disorder are grouped under the broad subclass of PDDs, that is, pervasive developmental disorder not otherwise specified (PDDNOS). Recently, the Work Group of the World Health Organization (WHO) proposed that in the International Classification of Diseases-10th edition (ICD-10), RS should be classified as a separate subcategory of PDDs. This proposal has given rise to a number of significant controversies. Some discussions have centered around the diagnostic validity of RS while others have focused on the suitability of classifying RS as a mental disorder as well as a subtype of PDDs. The WHO plans to publish the ICD-10 in 1993. The United

${ }^{\text {I}}$ The views expressed in this article are those of the author and do not represent the official positions of the DSM-IV Task Forces, Work Groups, or the American Psychiatric Association. 
States is under a treaty obligation with the WHO to maintain a coding and terminological consistency with the ICD system. The American Psychiatric Association Committee on Psychiatric Diagnosis and Assessment has already appointed several work groups to develop the DSM-IV and to publish it in 1993. While it is quite certain that the DSM-IV will continue the DSM-III-R concept of PPDs (Frances, Widiger, \& Pincus, 1989), it is uncertain at the present time whether DSM-IV will adopt ICD-10 concept of classifying RS as a subtype of PDDs.

The purpose of the present review is to examine the controversial issues and to provide constructive information which may be helpful to the PPDs Work Group of DSM-IV in making its final decision.

\section{ISSUE OF DIAGNOSTIC VALIDITY}

\section{Internal Validity}

Rett syndrome was originally described by Rett (1966), who reported (in German) his findings in 22 patients. However, it did not gain wide recognition until 1983, when a series of 35 cases from a pooled group of French-Portuguese-Swedish patients was reported in English (Hagberg, Aicardi, Dias, \& Ramos, 1983). Since then the International Rett Syndrome Association (IRSA) has been founded, international conferences on the syndrome have been held regularly, and at least four special medical journal issues have been devoted to the delineation and classification of RS: two in Japan and two in the United States (Hagberg, 1989).

Diagnostic criteria for RS were developed by a group representing the Centers for Disease Control, the American Association of University Affiliated Programs, and the IRSA (Trevathan \& Moser, 1988). This set of diagnostic criteria was developed based on the "Vienna Criteria" for the diagnosis of RS (Hagberg, Goutieres, Hanefeld, Rett, \& Wilson, 1985) and was adopted by a panel of international experts at the 1984 Rett Syndrome Conference. The diagnostic criteria contain three categories: necessary criteria, supportive criteria, and exclusive criteria. The necessary criteria include apparent normal prenatal and perinatal period; apparent normal psychomotor development through the first 6 months (in some cases development may appear to be normal for up to 18 months); normal head circumference at birth; deceleration of head growth between ages 5 months and 4 years; loss of acquired purposeful 
hand skills between ages 6 and 30 months; development of stereotypic hand movements such as hand wringing/squeezing, clapping/tapping, mouthing and washing/rubbing automatisms appearing after purposeful hand skills are lost; development of severely impaired expressive and receptive language; presence of apparent severe psychomotor retardation; appearance of gait apraxia and truncal apraxia/ataxia between ages 1 and 4 years; temporal social withdrawal; and diagnosis tentative until 2 to 5 years of age. Although "female sex" was considered by Hagberg et al. (1985) as one of the inclusion criteria of RS, the IRSA-CDC criteria do not include female sex as a necessary criterion. This decision was made with consideration of possible undiagnosed male cases (Trevathan \& Moser, 1988).

The supportive criteria of RS include breathing dysfunction, such as periodic apnea during wakefulness, intermittent hyperventilation, breathholding spells, and forced expulsion of air or saliva; EEG abnormalities including a slow-waking background and intermittent rhythmical slowing, epileptiform discharge, with or without clinical seizures; seizures; spasticity, often with associated development of muscle wasting; dystonia; peripheral vasomotor disturbances; scoliosis; growth retardation; and hypotrophic small feet. Although most RS patients display many of the supportive criteria, diagnosis is possible in the absence of all the supportive criteria, especially in young patients (Trevathan \& Moser, 1988).

The exclusion criteria of RS include evidence of intrauterine growth retardation; organomegaly or other signs of storage disease; retinopathy at birth; evidence of perinatally acquired brain damage; existence of identifiable metabolic or other progressive neurological disorder; acquired neurological disorders resulting from severe infections or head trauma. The presence of one or more of the exclusion criteria excludes the diagnosis of RS, regardless of whether all of the necessary criteria have been met in an individual patient (Trevathan \& Moser, 1988).

The IRSA-CDC diagnostic criteria for RS were restricted to include only typical patients, to ensure homogeneous patient population for future clinical and epidemiological research. Using both the Vienna criteria and the IRSA-CDC criteria, the estimated prevalence is at least $1: 10,000$ to 1:15,000, based on Swedish (Hagberg \& Witt-Engerstrom, 1987), Scottish (Kerr \& Stephenson, 1985), and Portuguese (Hagberg \& Witt-Engerstrom, 1987) studies. Today, RS is known to exist in all races and probably all countries. There are more than 1,500 recognized cases of RS worldwide. The many pathognomonic examples of its clinical expression have convinced clinicians of the existence of this unique syndrome. 


\section{External Validity}

In the past, children with Rett syndrome were frequently regarded as autistic (Olsson, 1987; Olsson \& Rett, 1985, 1987; Percy, Zoghbi, Lewis, \& Jankovic, 1988). Witt-Engerstrom and Gillberg (1987) have reported that $78 \%$ of children with RS have previously been diagnosed as having infantile autism. These reports raised a question in terms of the external validity of RS.

Several independent studies of behavioral observations demonstrated qualitative and quantitative differences between RS patients and persons with autism. Gillberg (1987) used a comprehensive questionnaire containing a number of semistructured questions and 130 items concerned with early characteristics of perceptual, motor, and emotional development in children. Eight mothers of eight young RS girls (ages 3-9 years) were interviewed using the questionnaire. Although there was considerable overlap between early symptoms in RS and infantile autism, there were indications that certain features might be used to differentiate the two disorders. For example, there were three items typical of autism that were rare in RS: "played only with hard objects," "did not like to be disturbed in her world," and "very pleased when left completely to herself."

Olsson and Rett (1985) compared the behavior of girls with RS to that of patients with infantile autism. Visual, acoustic, tactile, and gustatory stimuli and social contact were used for the comparison. The following are behaviors that were observed only in the RS patients and in no case of autism: slow movements plus hypoactivity; uniform stereotypic movements of hands with a broad-base stance; stereotypic washing movements of hands; stereotypic wetting of hands with saliva; stereotypic bringing together of hands; consistent, isolated stretching and flexing of the middle finger joints; episodic hyperventilation via mouth; and no chewing. Among behaviors characteristic of autism, the following five traits were not seen in the RS patients: predominant rejection of caressing and tenderness, conspicuous physical hyperactivity in terms of continuous grabbing and concomitant locomobility, excessive attachment to certain objects, rotation of small objects, and stereotypic playing habits. Thus, the authors had demonstrated that RS and autism could be differentiated based on behavioral observations.

Percy et al. (1988) analyzed motor and behavioral characteristics of 15 patients with RS. The authors found that children with RS differed from children with autism in having ataxia, breath-holding, hyperventilation, bruxism, simplicity of stereotypies, and hand apposition. On the other hand, the children with autism differed from those with RS in terms of overactivity, complex repetitive movements, and inappropriate vocalization. It is 
quite apparent that the course is different with RS progressing to various forms of neurological impairment that is not seen in autism.

Other variables such as etiology, outcome, and response to treatment have not yet been studied systematically in RS (Kerr, 1987; Moeschler, Charman, Berg, \& Graham, 1988). Nonetheless, available literature has generally demonstrated the extremely poor adult outcomes, the very marked motor impairment, and the development of scoliosis in RS patients. Thus, the course of RS is much different than that of autism, and the prognosis of RS is much worse than that of the autism. On the other hand, with increasing awareness of RS and its characteristic developmental profile (i.e., by 3 to 4 years of age, children with RS should have demonstrated the typical clinical features of RS), RS children should be easily distinguishable from those with autism (Trevathan \& Naidu, 1988).

Burd, Fisher, and Kerbeshian (1989) have argued that both RS and Heller syndrome have a phase of deterioration or disintegration, hence they should be classified together in a category of "pervasive disintegrative disorders." There is, however, difference in developmental profile (i.e., in RS the normal development usually presents through the first 6 to 12 months whereas in Heller syndrome the regression usually begin at age 3 or 4 years), clinical features (e.g., hand wringing/clapping/washing stereotypies in RS), and course (e.g., in RS there is gait apraxia and truncal apraxia/ataxia whereas in Heller's syndrome the motor function is usually normal).

Recently, Chatterjee et al. (1990) did two blind studies on the plasma glycosphingolipids in patients with the RS, other developmental disorders, and normal individuals from Baltimore (USA), Vienna (Austria), and Rostock (East Germany). The presence of an unusual glycosphingolipid was found in $70 \%$ of the patients with RS, and in approximately $10 \%$ of the patients with other disorders. However, this glycosphingolipid was absent from the plasma of normal controls.

These data suggest that RS has enough distinguishing features to warrant a distinct diagnostic entity.

\section{IS RETT SYNDROME A NEUROLOGICAL DISORDER OR A MENTAL DISORDER?}

Since RS is usually associated with a clear developmental regression, acquired microcephaly, ataxia, seizures, and pyramidal tract signs, there has been the argument that RS is a degenerative neurological disorder, hence it should not be classified as a mental disorder. 
It has long been recognized that damage or disease of the human brain (i.e., central nervous system) may result in impairments in both cognitive and behavioral functions. For example, a patient with a frontal-lobe tumor may appear apathetic and disheveled. As medical science advances, neurology has expanded its emphasis beyond structural components of disease to focus on neurochemistry, neuroendocrinology, and electrophysiologic disorders of the central nervous system while becoming more attentive to the behavioral and psychological aspects of such disorders. In an editorial entitled Whither Neurology? Martin (1984) defined the responsibility of neurologist as "the diagnosis and management of any disorder in which the involvement of the central nervous system is included in the differential diagnosis [which could, if the neurologist is interested, encompass] the diagnosis and management of disorders affecting behavior [italics added]."

Psychiatry, on the other hand, has become more biologically oriented, incorporating genetic, developmental, structural, neurophysiologic, neurochemical, and neuroendocrinologic knowledge of nervous system functionings. More and more psychiatrists now ask, in each type of mental disorder, to what degree is this biologic process determined by genetic and developmental factors, to what degree is it due to infectious or toxic agents, and to what degree is it socially determined? Generally speaking, it is not difficult in delineating a neurological and a psychiatric component in many neurological disorders accompanied by a psychiatric disorder (e.g., seizure disorder with substance abuse). In two situations such delineation may be difficult: (a) The same symptoms and signs can be construed either in a neurological or in a psychiatric way, for example, complex tics can be seen either as a neurological movement disorder or compulsions from a psychiatric frame of reference; and (b) syndromes/disorders with a characteristic mixture of neurological and psychiatric symptoms, for example, in the frontal-lobe tumors, the neurological signs are usually accompanied by slowing of mental activity and personality changes.

Nevertheless, it is obvious that neurology and psychiatry are each involved in the study and care of disorders of the central nervous system. The two disciplines have come together increasingly because the recognition of the common ground they share in dealing with many behavioral and emotional problems of human beings. There is emerging thought of reunion of neurology and psychiatry, making it a new discipline called "clinical neuroscience" (Detre, 1987). Therefore, any attempt toward developing a definition to delineate the precise boundaries between neurological/physical disorders and mental/psychiatric disorders would be a countermovement. On the other hand, including "neurological disorders with behavioral and emotional problems" such as autism and Tourette dis- 
order in the mental disorders classifications of ICD-10 as well as in the DSM-III-R takes us one step closer to developing a new discipline that will consider the "whole person" - not just the body or the mind. For the same reason, Rett syndrome should be included in the psychiatric nomenclature.

\section{SHOULD RETT SYNDROME BE CONSIDERED AS A SUBTYPE OF PERVASIVE DEVELOPMENTAL DISORDERS?}

There are clinicians who question that if many other degenerative nervous disorders (e.g., metachromatic leukodystrophy, Alexander's disease, Cockayne's syndrome) are not considered as subtypes of PDDs, then why is only RS being considered as a subtype of PDDs? They also argue that RS should be viewed as just one of the many neurological disorders that causes the general diagnostic category called "mental retardation," that is, RS should not be given a separate diagnostic category.

The first step of reviewing this issue is to study the definition of PDDs as defined by the DSM-III-R and proposed by the ICD-10. The PDDs are described as a group of mental disorders characterized by "qualitative impairments in 1) the development of reciprocal social interaction; 2) the development of verbal and nonverbal communication skills; and 3) imaginative activity. Often, there is a markedly restricted repertoire of activities and interests, which frequently are stereotyped and repetitive" (APA, 1987). These qualitative abnormalities are a pervasive feature of the individual's functioning in all situations, although they vary in degree. In most cases development is abnormal from infancy and, with only a few exceptions, the conditions are manifested from the first 5 years. It is usual, but not invariable, for there to be some degree of general cognitive impairment but the disorders are defined in terms of behavior that is deviant in relation to the individual's mental age (whether retarded or not) (WHO, ICD-10 draft, 1988).

The second step is to examine whether RS contains the clinical characteristics of PDDs. First, it is essential to know that RS is a progressive neurological disorder and there is variability of clinical presentation which depends on patient age and the disease stage. Cross-sectional findings at a single examination easily can lead to erroneous diagnostic association when the developmental profile is not analyzed (Hagberg, 1989). In classic RS, Hagberg and Witt-Engerstrom (1986) proposed a four-stage model: (a) Early onset stagnation stage is present between 6 months to $11 / 2$ years of age; (b) rapid developmental regression stage usually appears at 1-2 years of age; (c) pseudostationary stage usually occurs at 3-4 years of age, 
but can be delayed, and persists for many years or even decades; and (d) late motor deterioration stage often occurs during school age or early adolescence. Many investigators reported "autistic features" developed during Stage II (i.e., rapid developmental regression stage). The features include no sustained interest in persons or objects; stereotypic responses to environmental stimuli; absent or very limited interpersonal contact; manifestation of great anxiety and apparent fear when confronted with an unfamiliar situation, or even without evident stimulation; loss of already acquired elements of language; stereotypic hand movements included especially handwashing movements in front of the mouth or chest and rubbing motions of the hands; and repetitive blows on the teeth, grabbing of the tongue, and other movements (Hagberg et al., 1983).

At the early stage of RS, there are impairments in reciprocal social interaction and in patterns of communication. There is also manifestation of stereotypic behaviors. These are all the essential behavioral characteristics of PDDs. However, it is unclear whether these behaviors of RS are deviant in relation to the individual's mental age. While it is quite clear that all the RS patients continue to function in the range of severe-to-profound retardation, none of the RS studies has provided information regarding the relationship between the mental age and the level of social and language/speech developments/impairments. Without such information, it is difficult to differentiate the "impairment of social interaction" in the RS patients from the "lack of appropriate play and social skills" usually noticeable in young preschool and primary-aged children with moderate or severe retardation. It is also difficult to refute the argument that the stereotyped hand movements in the RS patients are similar to the stereotyped self-stimulating behaviors commonly seen in persons with severe or profound mental retardation.

Another issue here is the course of RS and its relationship with PDDs. While Rutter (personal communication, 1990) noted that the older RS patients continued to exhibit "autistic features," there were a few studies that reported the autistic features of RS which appeared in late infancy tended to diminish or resolve later at Stage III (i.e., pseudostationary stage). Autistic traits became less prominent in most of the patients followed until at least age 15 years (Hagberg, 1989; Hagberg et al., 1983). The ICD-10 draft also described that "social interest tends to be maintained" and that "often social interactions develop later." Gillberg (1987) reported that eye contact improved and sometimes became very intense. The RS girls did not mind human interaction and did not usually protest if their world was "disturbed." The automatisms also tended to become slower and more simplified in adulthood. In fact, the IRSA-CDC criteria define the communication dysfunction and social withdrawal as "temporal" 
features. It is unclear whether temporal means that all the RS patients eventually regain their communicative and social skills that match their mental age. In other words, it is unclear whether the RS patients at the late motor deterioration stage present a clinical picture commonly seen in immobile nonautistic persons with severe/profound mental retardation, or a picture seen in the patients who have improved/residual PDDs. Since RS is a newly recognized disorder, many questions remain concerning the course of RS. Further follow-up studies are needed to solve the questions.

\section{CONCLUSION}

The present literature review found that more than 200 articles on RS have been published by professional journals in the last 10 years. Several sets of diagnostic criteria for RS have been established prior to the development of the CDC-IRSA Criteria for RS, which is now widely used. Although the interclinician agreement (i.e., reliability) has not yet been studied systematically, the fact is that more than 1,500 identified cases, using the aforementioned criteria, have provided strong support for the internal validity of RS. Furthermore, several independent studies have demonstrated external validity of RS. All of this points to a compelling case for a distinct identification of RS.

There is convincing evidence showing that RS contains autistic features (i.e., impairments in social interactions and patterns of communication, and stereotypic behaviors). This is particularly true during the early stage of RS. However, due to a lack of empirical data, the question remains of whether these impaired functions and problematic behaviors of RS are deviant in relation to the individual's mental age, particularly in the older patients. Hence, it is not entirely clear whether RS has clinical features that meet the criteria for PDDs. Nevertheless, there are many reports that children with Rett syndrome were frequently regarded as autistic (Percy et al., 1988; Olsson \& Rett, 1987; Witt-Engerstrom \& Gillberg, 1987). Many clinicians tend to be impressed by the autistic features of their RS patients instead of by their "mental retardation features." On the other hand, though the autistic features of RS have been described as temporal features, there are not enough data to confirm that the older patients of RS suffer only severe or profound mental retardation. The possibility remains that the clinical manifestations of the older patients represent the continuum of the severer form of PDDs developed at the early stage of RS.

Thus, on the basis of available empirical data, it would be extremely unsatisfactory to bury the RS in an insufficiently defined manner as it is in DSM-III-R, that is, to view RS as one of many medical conditions as- 
sociated with autism or atypical autism. If we are to learn more about the relationship between RS and PDDs, it is critical that RS be clearly identified to enable it to be studied reliably.

Last, should the PDDs Work Group of DSM-IV decide to adopt RS as a subtype of PDDs, it should not adopt "apparent normal prenatal and perinatal period; apparent normal psychomotor development through the first 6 month" as necessary criteria for RS. Kerr and Stephenson (1985) had demonstrated distinct abnormalities of movement and indications of cognitive impairment before regression. DSM-IV should also consider the inclusion of the "Atypical RS" as another subtype of PDDs. Recent reports of patients who did not satisfy all the official criteria but present several features suggestive of RS, especially hand stereotypies, autism, and mental retardation add further foundation toward this decision. These cases did not meet the criteria because they either lacked a history of normal development for several months followed by a period of definite deterioration, or they had congenital or acquired encephalopathy of known cause (Goutieres \& Aicardi, 1986, 1987). However, these cases do seem to present cross-sectional features that are seen in PDDs. A controversial term of "Forme Fruste RS" has been used by many clinicians to describe the atypical/abortive variants of RS. It is to be expected that more cases with variant forms of RS would be forthcoming.

\section{REFERENCES}

American Psychiatric Association. (1987). Diagnostic and statistical manual of mental disorders (3rd ed., rev.). Washington, DC: Author.

Burd, L., Fisher, W., \& Kerbeshian, J. (1989). Pervasive disintegrative disorders: Are Rett syndrome and Heller dementia infantilis subtypes? Developmental Medicine and Child Neurology, 31, 609-616.

Chatterjee, S., Ghosh, N., Goh, K. M., Rohmann, E., Killian, W., \& Rett, A. (1990). Glycosphingolipids in patients with the Rett syndrome. Brain Development, 12, 85-87.

Detre, T. (1987). The future of psychiatry. American Joumal of Psychiatry, 144, 621-625.

Frances, A. J., Widiger, T. A., \& Pincus, H. A. (1989). The development of DSM-IV. Archives of General Psychiatry, 46, 373-375.

Gillberg, C. (1987). Autistic syndrome in Rett syndrome: The first two years according to mother reports. Brain Development, 9, 499-501.

Goutieres, F., \& Aicardi, J. (1986). Atypical forms of Rett syndrome. American Journal of Medical Genetics, 24, 184-194.

Goutieres, F., \& Aicardi, J. (1987). New experience with Rett syndrome in France: The problem of atypical cases. Brain Development, 9, 502-505.

Hagberg, B. A. (1989). Rett syndrome: Clinical peculiarities, diagnostic approach, and possible cause. Pediatric Neurology, 5, 75-83.

Hagberg, B. A., Aicardi, J., Dias, K., \& Ramos, O. (1983). A progressive syndrome of autism, dementia, ataxia, and loss of purposeful hand use in girls: Rett's syndrome: Report of 35 cases. Annals of Neurology, 14, 471-479.

Hagberg, B., Goutieres, F., Hanefeld, F., Rett, A., \& Wilson, J. (1985). Rett syndrome: Criteria for inclusion and exclusion. Brain Development, 7, 372-373. 
Hagberg, B. A., \& Witt-Engerstrom, I. (1986). Rett syndrome: A suggested staging system for describing impairment profile with increasing age toward adolescence. American Journal of Medical Genetics, 24, 47-59.

Hagberg, B. A., \& Witt-Engerstrom, I. (1987). Rett syndrome: Epidemiology and nosologyProgress in knowledge 1986-A conference communication. Brain Development, 9, 451-457.

Kerr, A. M. (1987). Report on the Rett Syndrome Workshop: Glasgow, Scotland, 24-25, May 1986. Journal of Mental Deficiency Research, 31, 93-113.

Kerr, A. M., \& Stephenson, J. B. P. (1985). Rett's syndrome in the west of Scotland. British Medical Journal, 291, 579-582.

Martin, J. B. (1984). Whither neurology? New England Journal of Medicine, 311, 1048-1050.

Moeschler, J. B., Charman, C. E., Berg, S. Z., \& Graham, J. M., Jr. (1988). Rett syndrome: Natural history and management. Pediatrics, 82, 1-10.

Olsson, B. (1987). Autistic traits in the Rett syndrome. Brain Development, 9, 491-498.

Olsson, B., \& Rett, A. (1985). Behavioral observations concerning differential diagnosis between the Rett syndrome and autism. Brain Development, 7, 281-289.

Olsson, B., \& Rett, A. (1987). Autism and Rett syndrome: Behavioral investigations and differential diagnosis. Developmental Medicine and Child Neurology, 29, 429-441.

Percy, A. K., Zoghbi, H. Y., Lewis, K. R., \& Jankovic, J. (1988). Rett syndrome: Qualitative and quantitative differentiation from autism. Journal of Child Neurolog, 3 (Suppl.), S65S67.

Rett, A. (1966). Uber ein eigenartiges hirnatrophisches Syndrom bei Hyperammoniamie im Kindesalter. Wiener Medizinische Wochenschrift, 116, 723-738.

Trevathan, E., \& Moser, H. W. (1988). Diagnostic criteria for Rett syndrome. Annals of Neurology, 23, 425-428.

Trevathan, E., \& Naidu, S. (1988). The clinical recognition and differential diagnosis of Rett syndrome. Journal of Child Neurology, 3 (Suppl.), S6-S16.

Witt-Engerstrom, I., \& Gillberg, C. (1987). Rett syndrome in Sweden. Joutnal of Autism and Developmental Disorders, 17, 149-150.

World Health Organization. (1988). International classification of disease (loth ed., draft). Geneva: Author. 